\title{
The Orientation of Unsymmetrical Molecules at Interfaces
}

\author{
S. H. Bauer and A. Pollack, George Herberl Jones Laboralory, The University of Chicago
}

(Received April 22, 1935)

\begin{abstract}
A function giving the distribution of the angles of orientation of the molecular axes from the normal to the interface can be obtained if one makes use of a number of special assumptions regarding the forces of orientation. This is compared with the distribution calculated on assuming an extreme form of Langmuir's principle of independent surface action. The importance of having such a function is pointed out.
\end{abstract}

$S^{\mathrm{I}}$ INCE the publication of the often quoted paragraph by W. B. Hardy in $1912^{1}$ on the orienting effects of unsymmetrical fields of molecules at interfaces, much work has been done in this direction which has led many to state that in cases of saturated films close to one hundred percent orientation exists. Such estimates were made on the basis of qualitative reasoning ${ }^{2}$ as well as on quantitative calculations. ${ }^{3}$ An obvious objection against the theory of surface orientation, sometimes presented as being of great importance while at other times minimized, is that the random motion of the molecules due to their thermal agitation will serve to overcome the orienting forces. ${ }^{4}$

The purpose of this paper is to present a more or less quantitative estimate of this effect based on the classical principles of statistics and some of the present day concepts of oriented dipole layers, it being expressly understood that the picture used is a highly artificial one.

From a kinetic theory point of view, one must consider a distribution in orientation so that an "oriented layer" really stands for a more or less defined layer of molecules whose molecular axes make an average angle with the surface which is not zero. In the case where the distribution in the inclination from the normal to the surface alone is to be considered, the generalized Boltzmann

\footnotetext{
I Hardy, Proc. Roy. Soc. A86, 634 (1912).

¿ Harkins, Alexander's Colloid Chemistry; Chem. Catalog Co., 1926, vol. 1, p. 192 .

3 langmuir, Colloid Symposium Monograph III; Chem. Catalog Co., 1925, p. 48ff., who makes use of the principle of independent surface action, found the probability that a molecule like methyl alcohol should have its electrical axis oriented normally to the alcohol-vapor interface with its $\mathrm{OH}$ group in the alcohol phase is 300 to $10^{5}$ times as great as the probability of orientation in the opposite direction.

1 Compare: Langmuir, reference 3 , p. 54 and Adam, The Physics and Chemistry of Surfaces; Oxford, 1930, pp. 146, 150 .
}

function reduces to the well-known expression

$$
d n=C e^{-V(\theta) / k T} \sin \theta d \theta,
$$

where $\theta$ is the inclination of the molecular axis from the $Z$ axis (taken normal to the surface); $d n$ is the number of molecules between the angles $\theta$ and $\theta+d \theta ; V(\theta)$ is the potential energy of orientation of the molecule at $\theta$; and $C$ is a constant, containing among other factors, the a priori probability of a given orientation.

If it is assumed that $C$ is independent of $\theta$ (which may not be true for interfaces where the molecules are crowded closely together so that the $a$ priori probability will depend on geometric factors), it becomes obvious from (1) that

$$
\begin{aligned}
& (s-1) \int_{\theta_{1}}^{\theta_{2}} e^{-V(\theta) / k T} \sin \theta d \theta \\
& =\int_{0}^{\theta_{1}} e^{-V(\theta) / k T} \sin \theta d \theta+\int_{\theta_{2}}^{\pi} e^{-V(\theta) / k T} \sin \theta d \theta,
\end{aligned}
$$

where $1 / s$ is the fraction of molecules oriented between $\theta_{1}$ and $\theta_{2}$. On the other hand, assuming random orientations, that fraction can be calculated from

$$
1 / s=\frac{1}{2}\left(\cos \theta_{1}-\cos \theta_{2}\right) .
$$

In (2), $1 / s$ can readily be found as soon as $V(\theta)$ is known. By extending the method of Langmuir ${ }^{5}$ this function can be obtained directly; whence the distribution function. Further, on differentiating $V(\theta)$ one can get some idea of the type of force which one must imagine to exist near and at the interface in order to explain the orienting effects. This method will be illustrated in the latter part of the paper.

One can, however, approximate $V(\theta)$ by assuming a field of force as a function of $\theta$ of a

\footnotetext{
${ }^{5}$ Reference 3, p. 50.
} 
form which would be expected under these circumstances. If this were to result in an expression for $V(\theta)$ containing one arbitrary constant, its value can be estimated from the average potential energy of orientation per molecule which can be obtained experimentally; thus

$$
\bar{v}=\frac{C^{1} \int_{0}^{\pi} e^{-V(\theta) / k T} V(\theta) \sin \theta d \theta}{C^{1} \int_{0}^{\pi} e^{-V(\theta) / k T} \sin \theta d \theta} .
$$

It is true that differences of opinion exist as to what experimental quantity should be used as a measure of $\bar{v}$ but fundamentally they are very much alike.

Harkins considers, as will be done in this discussion, the energy of adhesion, defined as

$$
\begin{aligned}
E_{A} & =\left(\gamma_{1}-T \frac{\partial \gamma_{1}}{\partial T}\right)+\left(\gamma_{2}-T \frac{\dot{\partial} \gamma_{2}}{\partial T}\right)-\left(\gamma_{i}-T \frac{\partial \gamma_{i}}{\partial T}\right) \\
& =E_{1}+E_{2}-E_{i}
\end{aligned}
$$

where $\gamma$ represents the free surface energy and $E_{1}, E_{2}, E_{i}$, the total surface energies of the liquids 1,2 , and of the interface between them, respectively. $E_{A}$, then, is the total energy necessary to tear apart a column one square centimeter in cross section of liquid 1 from a similar column of liquid 2 when the ends of the columns are in contact.

It can be readily seen from the available data that $E_{1}$ for organic liquids, particularly for those of aliphatic nature, differ very little from one another even when some are of the "polar" type, whereas such a difference is markedly shown, when the second liquid is water, in $E_{i}$. This must be due to the fact that in cases where $E_{i}$ is small (i.e., when $E_{A}$ is large) the polar groups of the organic molecules in the interface are solvated in the water phase. ${ }^{b}$ Hence the difference between the $E_{A}$ for a hydrocarbon on water and that for the corresponding acid, aldehyde, alcohol, etc., on water is a measure of the potential energy of orientation per $\mathrm{cm}^{2}$ of surface. ${ }^{7}$ We can now write

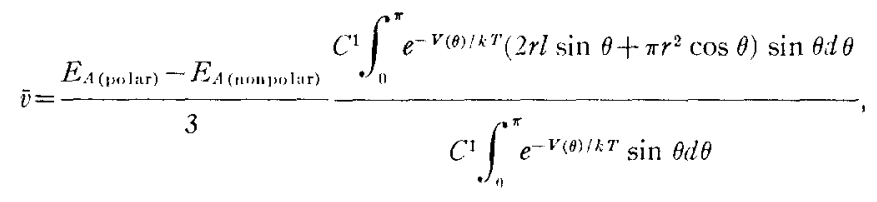

where the second factor of the right member is the average area of a cylindrical molecule of length $l$ and radius $r$ projected on the interface while the first factor gives the potential energy of orientation per $\mathrm{cm}^{2}$ of interface for the polar molecules. The factor 3 in the denominator is arbitrary but can be justified on the ground that $\left(E_{A \text { (polar) }}-E_{A \text { (nonpolar })}\right)$ is the potential energy per $\mathrm{cm}^{2}$ of three processes of orientation, viz.,

(a) Change in the orientation of the water molecules in the interface as the bounding field changes from that due to the polar ends of organic molecules to that produced by water vapor;

(b) Disorientation of the organic molecules in the interface-from the polar ends in the water phase to that of a ranclom distribution;

(c) Finally, as the water surface is sufficiently removed, there takes place another orientation in the organic liquidvapor interface so that the nonpolar ends extend toward the vapor phase. Since we are interested in the energy required for step (b) only, and further, since it is generally conceded that as much as 94 percent of the free surface energy is localized in the first layer of molecules, ${ }^{8}$ the factor $\frac{1}{3}$ cannot be greatly in error. The form of the resulting distribution function will certainly be little affected.

${ }^{6}$ Harkins presents this view very clearly: reference 2 , p. 208. "If a bar of . . . octyl alcohol of unit cross section is pulled in two at a plane, the molecules should first orient themselves on both sides of the plane where the break is about to occur. . . The final work of separation will be effect ed most largely by the attraction between the nonpolar ends of the atcohol molecules. . . Thus it would be expected that the wort of separation of octyl alcohol from itself would be not greatly higher than the work of separation of octane from octane. . . If however, the octyl alcohol is pulled a way from water, the relations are different. The hydroxyl groups should be turned toward the water. . . The final break must come between the polar $\mathrm{OH}$ groups of the alcohol and the water. Therefore the work of adhesion should be high."

"For data which may be used in these calculations see Harkins and Ewing, J. Am. Chem. Soc, 42, 2539 (1920); Harkins and Cheng, ibid. 43, 35 (1921); Silberstein, Zeits. f. physik. Chemie A143, 157 (1929); Satterly and Colling. wood, Trans. Roy. Soc. Can. 25 (III), 205 (1931); Int. Crit. Tab. 4, p. 450 ff. compiled by Harkins and Young.

${ }^{8}$ Edser, Fourth Colloid Report, 1922, p. 58. 
Langmuir employs in his calculations differences in the $E_{i}$ term only. ${ }^{9}$ It is obvious, however, that this method is a close approximation to that of Harkins since the $E_{1}$ 's differ very little for most organic substances. Adam, ${ }^{10}$ on the other hand, suggests that the latent heat term $(-T \partial \gamma / \partial T)$ should not be taken into account in these considerations.

At any rate, on equating expressions (4) and (6), one can solve for $V(\theta)$ and for the unknown constant whatever value one uses for the average energy of orientation per unit area. To illustrate this procedure for obtaining the distribution function and to compare with similar results derived by extending Langmuir's method as indicated above, we shall outline the calculations that were carried through for the case of octyl alcohol-water interface. The molecules are assumed to remain linear ${ }^{13}$ and the force function is taken to be independent of $\theta$, i.e., is a constant $F_{0}$ in a direction normal to the interface. Then obviously

$$
V(\theta)=-M \cos \theta
$$

where $M$, depending on $F_{0}$ and the length of the molecule, is the unknown constant to be determined by equating (4) and $(6) ; V(\pi / 2)=0$ is here taken as the reference of potential energy. On substituting in the resulting equation $\cos \theta=x$ and $M, k T=a$ as is usually done, one gets

$$
\begin{array}{r}
a k T \int_{+1}^{-1} e^{a x} d x=-p \int_{+1}^{-1} e^{a x}\left(2 r l\left(1-x^{2}\right)^{\frac{1}{2}}\right. \\
\left.+\pi r^{2} x\right) d x
\end{array}
$$

where $p=\left(E_{A(P)}-E_{A(N P)}\right) / 3$ and the unknown to be solved for is now $a$.

The right member of (8) can be integrated only when $e^{a x}$ is expanded into a series. On performing the integration and simplifying, and at the same time also expanding the integral of the left member into a similar series for computational purposes, there results the relationship

$$
\begin{aligned}
& p r l \pi\left(\frac{1}{2}+\frac{a^{2}}{16}+\frac{a^{4}}{384}+\frac{5 a^{6}}{9216}+\cdots+\frac{a^{n}}{n ! 2 \cdot 4 \cdot 6 \cdots(n-2) n(n+2)} \frac{3 \cdot 5 \cdot 7 \cdots(n-1)}{2 \cdot \cdots)}\right. \\
& =k T\left(\frac{a^{2}}{3}+\frac{a^{4}}{30}+\frac{a^{8}}{840}+\frac{a^{8}}{45360}+\cdots \frac{n a^{n}}{(n+1) !} \cdots\right)+p \pi r^{2}\left(\frac{a}{3}+\frac{a^{3}}{30}+\frac{a^{5}}{840}+\frac{a^{7}}{45360}+\cdots+\frac{m a^{m}}{(m+1) !} \cdots\right),
\end{aligned}
$$

where $n$ is an even number only, and $m=n-1$. These series, although absolutely convergent for all values of $a$, do not converge very rapidly in this particular case, so that it was necessary to obtain the needed root of an eighth degree equation. This was done by a method of successive approximations and we proved satisfactorily that further terms in the series will affect the result by less than 2 percent.

\footnotetext{
"Values used by that writer are given in Alexander, reference 2, D. 536, and Colloid Symposium Monograph, reference 3, p. 53 . See also work of Snyth, I. Am. Chem. Soc. $51,2646,2660(1929)$.

to $\mathrm{His}$ book, reference $4, \mathrm{p} .151$.

11 This is a point of departure from the ideas expressed by Langnuir (Colloid Symposium, III) where he considers the molecules of long chained fatty acids and alcohols in such a position in the interface as to keep their polar ends anchored in the interface where they can behave as a two-dimensional gas while the rest of the chain twists and flips about in the body of the liquid. For octyl alcohol, this effect is probably not very important.
}

The actual data used were: ${ }^{2}$

$E_{A(P)}=164.6 \mathrm{ergs} / \mathrm{cm}^{2}$ for $n$-octyl alcohol at $20^{\circ} \mathrm{C}$ on water

$E_{A(N P)}=107.4 \mathrm{ergs} / \mathrm{cm}^{2}$ for $n$-octane alcohol at $20^{\circ} \mathrm{C}$ on water

$\pi y^{2}=21.6 \times 10^{-1 \mathrm{~s}} \mathrm{~cm}^{2}$

$2 \pi r l=6.29 \times 10^{-16} \mathrm{~cm}^{2}$

$1 / k T=2.49 \times 10^{13}$

giving

$a=2.54$,

from which the distribution function (1) was calculated. Curve 1 of Fig. 1 is a plot of the values of $1 / \mathrm{s}$ as calculated from (3) to be expected assuming random orientation, while curve 2 is the plot of the above calculated distribution (Eq. (2)) assuming a constant force acting normal

\footnotetext{
${ }_{12}$ The value of $r$ was obtained from the area at zero compression (of film balance) for the aliphatic alcohols The length was estimated from the molecular volume and value of $r$, as calculated from the density $(=0.827)$ at $20^{\circ} \mathrm{C}$.
} 


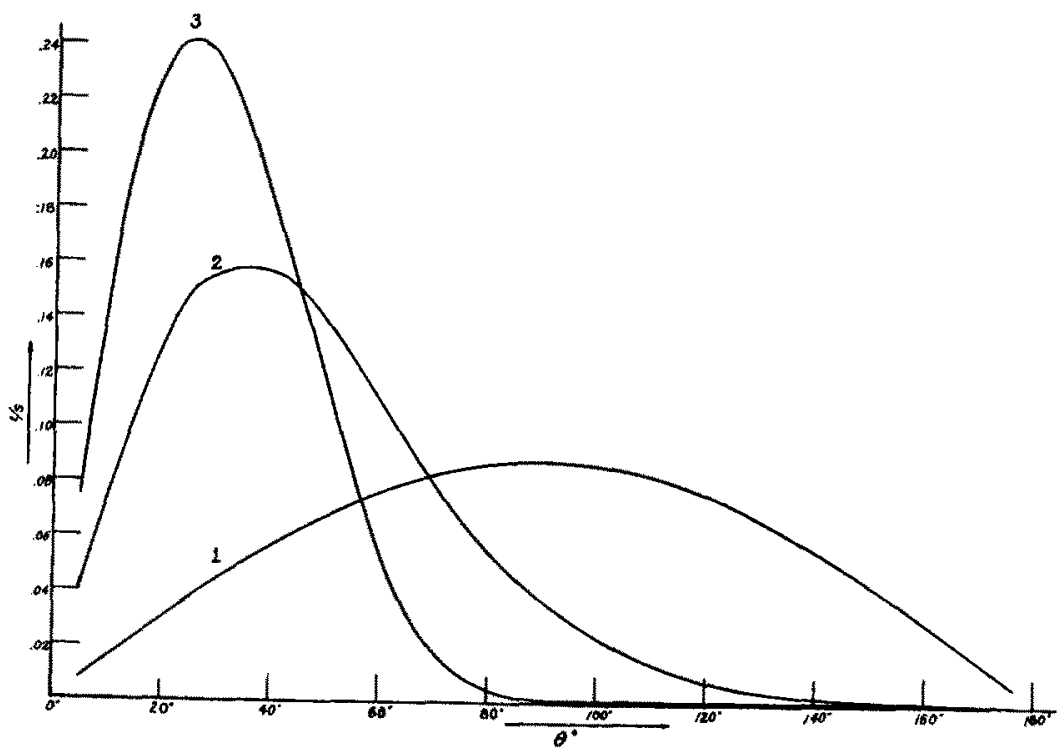

FIG. 1.

to the interface and of magnitude consistent with the measured potential energies of orientation.

We now extended Langmuir's principle of independent surface action to an exaggerated degree by calculating the interfactial energy an actyl alcohol molecule has when it is oriented at different angles from the normal to the interface. Fig. 2 best illustrates the method used. The $X Y$ plane represents the water-alcohol interface; the cylinder represents the molecule oriented at an angle $\theta$ from the $Z$ axis; the section of the cylinder above the plane $C H F G$ is supposedly the polar end while that below the plane, the nonpolar end. It was then a simple geometrical problem to calculate the areas and their projections on the $X Y$ plane of the polar section in contact with the water and alcohol phases; similarly for the nonpolar section. The values of the areas at a given angle were then multiplied by their respective interfacial energies and the products added to give the total energy of orientation at that angle. Again, $V(\pi / 2)$ was taken as the zero of potential energy. From the resulting function $V(\theta)$, curve 3 in Fig. 1 was obtained using Eq. (2).
The geometrical constants needed in the calculations were rather arbitrarily estimated from the ratios of the lengths of the $\mathrm{OH}$ and $\mathrm{CH}_{2}$ groups as obtained from $\mathrm{x}$-ray data ${ }^{18}$ and our previous values of $l$ and $r$. Thus

$$
\begin{array}{ll}
r=2.62 \times 10^{-3} \mathrm{~cm}, & l_{\text {OE }}=2.5 \times 10^{-8} \mathrm{~cm} \\
l=12.0 \times 10^{-3} \mathrm{~cm}, & l_{\mathrm{C}_{3} \mathrm{H}_{8}}=9.5 \times 10^{-3} \mathrm{~cm}
\end{array}
$$

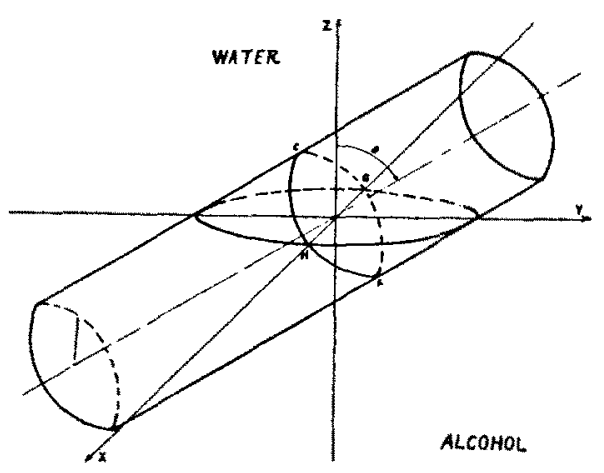

FIG. 2.

${ }^{18}$ Clark, Applied X-Rays; McGraw-Hill Co., New York, 1932, p. 319ff. 
The four interfaces to be considered and their respective energies as estimated by Langmuir from such data as the heats of evaporation and the partial pressures of binary mixtures are: ${ }^{14}$

\begin{tabular}{ll} 
& \multicolumn{1}{c}{$E_{i}$} \\
$\mathrm{OH}$-water & $-12.6 \mathrm{ergs} / \mathrm{cm}^{2}$ \\
$R$-alcohol & +6.7 \\
OH-alcohol & +24.4 \\
$R$-water & +37.4
\end{tabular}

To say the least, the authors are convinced that little weight can be placed on such an analysis until many calculations of a similar nature have been carried through. It is to be noticed that although in cases 2 and 3 the $V(\theta)$ functions differ markedly from one another and even more so do their corresponding force functions, the actual distributions in orientation are similar. Further, it is to be remembered that the conclusions reached apply only to gaseous films where the a priori probability of any orientation is independent of $\theta$. As has been indicated above, in cases where the film approaches the condensed state when various crystallizing forces tend to bring in such a dependence, the constant $C$ of (1) and of the subsequent equations would have to be expressed as an explicit function of the angle and made part of the integrand. The authors considered using a function which had maximal values at $\theta=0$ and $\pi$ to study the effect on the form of the distribution obtained. The

\footnotetext{
${ }^{14} \mathrm{He}$ does not give the values for $E_{\text {or-alcohol and }}$ $E_{R}$-alcohol. These were estimated from the energies $E_{R-\mathrm{OH}}, E_{R-R}$ and $E_{\mathrm{OR}-\mathrm{OH}}$ whose magnitudes he gives as $33.7,0$ and -12.6 , respectively, by taking into consideration the relative areas of the $\mathrm{OH}$ and $R$ parts of the alcohol phase presented to the molecule in the interface.
}

arbitrariness introduced by selecting such a function at random is evident, particularly so when Rideal and his co-workers are inclined to believe that the maximal values really exist at other angles than 0 and $\pi$. The estimation of the ratio of the magnitudes of the maxima to those of the minima would be difficult if at all possible.

We hesitate to discuss the significance of these particular distribution functions or of the approximate validity of the method used to calculate them. We do wish to indicate, however, that did valid methods exist by means of which such distributions could be calculated, the resulting functions would be used in all surfacechemistry discussions wherein "orientation" is assumed or implied. Outstanding experiments which would be particularly affected are the contact potential measurements due to unimolecular insoluble films spread on the surfaces of very dilute acid or alkali solutions. If the equation relating the potential difference due to the film with the average dipole moment of the molecules is approximately true, ${ }^{15}$

$$
\Delta V=2 \pi n \bar{m}
$$

the distribution functions obtained not only permit one to calculate the actual dipole moment but also predict exactly the effect of temperature on $\Delta V$ (provided the nature of the film itself is not changed by the alteration of temperature).

In conclusion, the authors wish to express the sincerest thanks to Professor W. D. Harkins for the numerous helpful discussions relating to the material of this paper.

\footnotetext{
w Harkins and Fischer, J. Chem. Phys. 1, 852 (1933).
} 